\title{
The Comparison of Clinical Assessment Tools for the Foot Posture
}

\author{
Jin-yi Lee ${ }^{1}$, BHSc, PT, Jong-duk Choi ${ }^{2}$, PhD, PT \\ ${ }^{1}$ Dept. of Physical Therapy, The Graduate School, Daejeon University, \\ ${ }^{2}$ Dept. of Physical Therapy, College of Natural Science, Daejeon University
}

\begin{abstract}
It is important to assess foot posture when investigating the relationship between lower extremity dysfunctions and foot types. Although several measurements of static foot posture have been used, there is no consensus regarding clinical measurements for foot posture. The aim of this study is to explore the differences among navicular drift (NDt), foot posture index (FPI), arch index (AI), dorsal arch height ratio (DAHR), normal navicular height truncated (NNHt) and to discover the most effective measurement. After foot types were classified by navicular drop test (NDp), clinical measurements of NDt, FPI, AI, DAHR, and NNHt were performed on 64 subjects' feet. ANOVA analysis was used for the variance of the difference between the NDp and the five kinds of clinical measurements, and the level of significance was set at $a=.05$. The results showed that all five clinical measurements demonstrated significant differences with navicular drop. In post-hoc, FPI and NNHt showed significant differences in all foot types. The five clinical measurements are suitable the classification of foot types through the NDp. Therefore, it could be possible to assess correct and objective foot posture by using FPI and NNHt.
\end{abstract}

[Jin-yi Lee, Jong-duk Choi. The Comparison of Clinical Assessment Tools for the Foot Posture. Phys Ther Kor. 2012;19(3):115-123.]

Key Words: Assessment tool; Foot posture; Pronated foot; Supinated foot.

\section{Introduction}

Abnormal foot posture, such as excessive pronation and supination, has been identified as a causative factor in the dysfunction of feet and the lower extremities (Allen and Glasoe, 2000). The height of the medial longitudinal arch (MLA) has been generally considered to be a factor that predicts injury (Williams and McClay, 2000). The MLA plays a role in absorbing shock. Foot types, bony structures, ligamentous stability, and muscular fatigue affect the height of the MLA. Arch height influences the motion of the lower extremities (Nigg et al, 1992; Nigg et al, 1993) and balance ability (Hertel et al, 2002) and abnormal arch height is associated with overuse injuries of the lower extremity (McCory et al, 1997). Thus, the clinical assessment of foot posture is the essential component for the management of foot dysfunction (Power et al, 1995).

Foot types are usually classified as pronated, normal, or supinated feet. Excessive pronation, which is characterized by the flattening of the MLA, causes hypermobility in the mid-foot. Excessive pronation increases the internal rotation of the tibia during the stance phase in gait cycle and alternates the position of the patella, which causes the subluxation of the patella and the imbalance of the quadriceps muscle (Brukner, 1996). An excessively supinated foot, known as a high-arched foot, causes hypomobility in the mid-foot and absorbs less shock. The movement of the supinated foot causes the lateral rotation of the femur and the tibia opposite to that of the pronated foot (Inman, 1969) and the rigid foot produce varus movement of the knee (Sahrmann, 2011).

Corresponding author: Jong-duk Choi choidew@dju.kr 
The navicular drop test (NDp) has been widely used in recent studies as a valid method for determining excessive pronation (Albensi et al, 1999) and has also been known as a method of predicting lower limb pathology (Bennett et al, 2001). The NDp, designed by Brody in 1982, is a method of measuring the vertical change of the navicular tubercle height from the bottom, which shows the amount of movement of the navicular bone in the sagittal plane (Reilly et al, 2009). It appears to be a simple and reliable measurement of foot posture (Shrader et al, 2005). Also, the NDp is a useful clinical measurement of overuse syndrome of the lower limbs. Therefore, in this study, NDp was performed to classify foot posture.

The navicular drift test (NDt) is a method of quantifying this medial-lateral movement of the mid-foot (Menz, 1998). Although there is no reliability study of this test, Menz (1998) suggested that the measurement of navicular drift provides an indicator of the medial prominence of the talonavicular joint when the foot moves from a neutral to a resting position.

The foot posture index (FPI), an observational scoring system has been developed on the basis of measuring positions of anatomical foot landmarks for assessing static foot posture (Redmond et al, 2006). It is a fast and simple method of classifying foot posture based on six different visual foot posture criteria, and it has the advantage of measuring the foot in multiple planes. However, it appeared to be poorly correlated with radiographic measurements (Scharfbillig et al, 2004).

The arch index (AI) was widely reported as a footprint measurement that is defined as the ratio of the total area of the foot, excluding the toes, to the related medial mid-foot area (Cowan et al, 1994). Although AI was considered to be an invalid clinical measurement to classify arch height (Hawes et al, 1992), recent study has shown significant correlations between AI and radiographs (Muller et al, 1993).

The dorsal arch height ratio (DAHR), designed by
Williams and McClay (2000), is an alternative to the NDp. It is a method of measuring the vertical change in dorsal arch height and the amount of foot mobility (Scharfbillig et al, 2004), and it showed high validity and reliability when compared to radiographs (McCrory, 1997).

The criteria validity of the normalized navicular height truncated test (NNHt) was established by the radiographic method (Williams and McClay, 2000) and the height measurement of the navicular has been shown to be an indicator of the mobility of the navicular (Cornwall and McPoil, 1999). However, as there is no generalized value, it is difficult to distinguish between normal and abnormal feet (Menz and Munteanu, 2005).

Although several clinical measurements have been described to classify and quantify static foot postures, the most useful and proper clinical measurement for assessing foot posture is not common. Also, the validity and the reliability of some measurements have been shown, but there have been few studies that compared each measurements to find out the more effective measurements. In addition, although some studies regarding reliability and validity with radiographs have been reported, there is no consensus on the validity and reliability of the measurements. There is little research on the validity of clinical measurements for assessing foot posture.

Thus, the aim of this study is to explore the differences among NDt, FPI, AI, DAHR, NNHt and to discover the most effective measurement.

\section{Methods}

\section{Subjects}

The feet of sixty-four subjects who were recruited randomly were measured and analyzed. After the $\mathrm{ex}^{-}$ aminer sufficiently explained the purpose and method of the study to the subjects, they agreed to take part in this study. Exclusion criteria included a history of congenital deformity in the lower extremities or feet, 


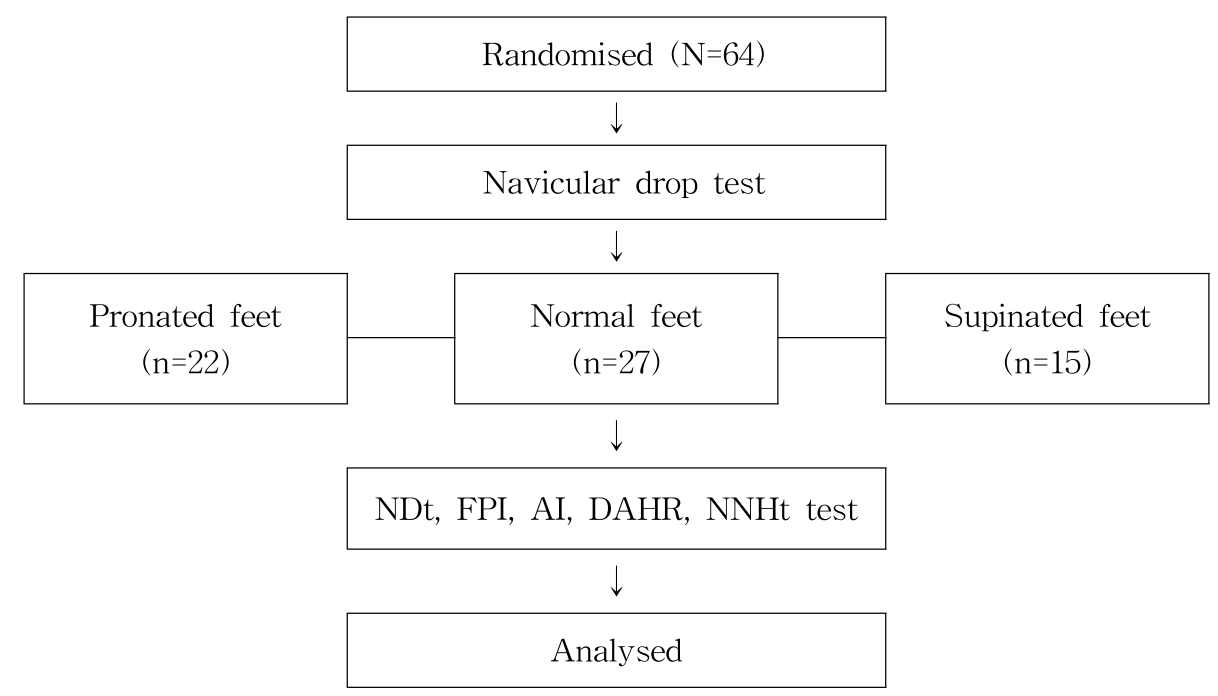

Figure 1. Flowchart of subjects through this study.

a history of fracture in the lower extremities or feet, a history of trauma or pain, and previous systemic disease that could affect lower extremity or foot posture.

The subjects of the study were common people between 15 and 51 years of age. The demographic characteristics of the subjects are summarized in Table 1. Subjects were classified as having pronated feet (22 feet), normal feet (27 feet), or supinated feet (15 feet) according to their foot postures.

\section{Clinical measurements}

1) Navicular drop test

In the current study, the NDp, which was described by Plisky et al (2007), was used to determine the sagittal plane displacement of the navicular between the resting and neutral stance positions $(\mathrm{ICC}=.78 \sim .83)$ (Muller et al, 1993). The participant was seated with both feet on the ground and his or her knees flexed at 90 degrees. The navicular tuberosity, the most medial aspect of the navicular was marked. The clinician palpated the medial and lateral aspects of the talus head with the thumb and index finger placed just in front of the anterior aspect of the fibula and just anterior and inferior to the medial mallelous. Neutral $\mathrm{po}^{-}$ sition of subtalar joint was identified by asking the subject to invert and evert the hindfoot and ankle while the examiner, using the thumb and the index, palpated the anterior medial and anterior lateral head

Table 1. General characteristics of subjects and navicular drop score

$(\mathrm{N}=64)$

\begin{tabular}{ccccc}
\hline \hline & $\begin{array}{c}\text { Pronated foot } \\
(\mathrm{n}=22)\end{array}$ & $\begin{array}{c}\text { Normal foot } \\
(\mathrm{n}=27)\end{array}$ & $\begin{array}{c}\text { Supinated foot } \\
(\mathrm{n}=15)\end{array}$ & $\boldsymbol{x}^{2} / \mathrm{F}$ \\
\hline Sex (male/female) & $3 / 19$ & $6 / 21$ & $5 / 10$ & 2.028 \\
Age (yrs) & $28.80 \pm 6.04^{\mathrm{a}}$ & $33.18 \pm 9.71$ & $30.26 \pm 9.69$ & 1.625 \\
Height $(\mathrm{cm})$ & $161.62 \pm 6.11$ & $163.61 \pm 7.05$ & $164.08 \pm 4.82$ & .881 \\
Weight $(\mathrm{kg})$ & $57.12 \pm 8.42$ & $60.60 \pm 11.62$ & $62.65 \pm 11.31$ & 1.330 \\
Shoes size $(\mathrm{mm})$ & $240.90 \pm 10.53$ & $242.96 \pm 11.95$ & $246.66 \pm 16.76$ & .907 \\
Navicular drop $(\mathrm{mm})$ & $11.26 \pm 1.67$ & $6.54 \pm 1.62$ & $3.18 \pm .68$ & $139.733^{\star}$ \\
\hline
\end{tabular}

${ }^{a}$ mean \pm standard deviation, ${ }^{*} \mathrm{p}<.05$. 
of the talus for congruency (Sell et al, 1994). With the foot in neutral position of subtalar joint, the clinician measured the distance between the navicular tubercle and the floor in millimeters by using a digital height gauge. We asked the subjects to relax bipedal stance into a full weight-bearing position, and the resulting position of the navicular was measured with a digital height gauge. We recorded the distance between the original height of the navicular and its final weight-bearing position as the subject's navicular drop score. We measured NDp three times by using mean values. The subjects were categorized into one of three groups: a normal foot (between 5 and $9 \mathrm{~mm}$ of navicular drop), an excessively pronated foot (more than $10 \mathrm{~mm}$ of navicular drop), and an excessively supinated foot (less than $4 \mathrm{~mm}$ of navicular drop) (Cote et al, 2005).

\section{2) Navicular drift test}

NDt is a measure of MLA mobility and quantifies the amount of horizontal medial navicular excursion between the positions of the subtalar joint when in the neutral sitting and relaxed standing postures (ICC=.31 .62) (Vinicombe et al, 2001). The NDt were obtained by marking the horizontal (medial) position of the navicular tuberosity while the participant sat positioned in neutral position of subtalar joint (Vinicombe et al, 2001). The participant was then asked to assume a relaxed standing position while the horizontal position of the navicular tuberosity was re-marked. The horizontal distance between the two marks was used to represent the medial drift of the navicular (Vinicombe et al, 2001). Measurements were obtained to the nearest millimeter using a ruler. A smaller NDt value represented a foot posture with a higher, less deformable arch. We measured navicular drift three times by using a mean value.

\section{3) Foot posture index}

The procedure for scoring of the FPI- 6 that has been described in the literature (Redmond et al, 2006). FPI is a system for observing and rating static foot posture, incorporating six criteria, with the subject standing in a relaxed bipedal position $(\mathrm{Kw}=.86)$ (Ferrari and Morrison, 2009). The six-variable (FPI-6) was used to characterize the static foot posture of each subject. While each subject stood in their relaxed stance position with their arms by their sides and looking straight ahead, each of the six clinical criteria of the FPI-6 were assessed and scored on a 5-point scale from -2 to +2 by the same individual. The results were combined, resulting in a summative score ranging from -12 (highly supinated) to +12 (highly pronated) with negative values representing a supinated posture and positive values characterizing a pronated position. Reference values as follows: normal $(0 \sim 5)$, pronated (6 9), highly pronated $(10+)$, supinated $(-1 \sim-4)$, highly supinated (-5 -12). In this study, modified scoring system was used as follows: normal $(0 \sim 5)$, pronated (6 12), supinated $(-1 \sim-12)$. The six criteria are as follows: the position of the head of the talus, the observation of the curves above and below the lateral malleoli, the extent of calcaneus inversion/eversion, the extent of the bulge in the region of the talonavicular joint, the congruence of the MLA, and the extent of the abduction/adduction of the forefoot on the rear-foot.

\section{4) Arch index}

AI of the static footprint was obtained by using imprint material, with the subject standing in a relaxed position (ICC=.99) (Menz and Munteanu, 2005). Using the AutoCAD ${ }^{1)}$ computer program, the $\mathrm{AI}$ was calculated as the ratio of the area of the middle third of the footprint to the entire footprint area. The higher the arch, the lower the AI (Cowan et al, 1994).

5) Dorsal arch height ratio

DAHR is the ratio of dorsum height relative to

1) AutoCAD 2010, Autodesk Inc., Seoul, Korea. 
the truncated length of the foot, excluding the toes. Measurements of DAHR followed the method $\mathrm{de}^{-}$ scribed in the literature ( $\mathrm{ICC}=.811$ ) (Williams and McClay, 2000). Firstly, total foot length was measured from the most posterior portion of the calcaneus to the end of the longest toe with a sliding ruler. Next, to determine the point of $50 \%$ of total foot length, the previously measured total foot length was divided in half, and the dorsum was marked at the $50 \%$ length point using a pen. Then, dorsal arch height was measured marked point from ground. Next, truncated foot length from the most posterior portion of the calcaneus to the center of the first metatarsophalangeal joint was measured. Both measurements were obtained using a sliding ruler, and the values were rounded to the nearest millimeter. The DAHR was calculated by dividing the dorsum foot height by the truncated foot length.

\section{6) Normalized navicular height truncated}

NNHt is the ratio of navicular height relative to the truncated length of the foot (ICC=.93) (Williams and McClay, 2000). First, with the subject standing in a relaxed position, the most medial prominence of the navicuar tuberosity was palpated and marked with a pen. Digital height gauge was then used to measure from the bottom to the top of the navicular tuberosity. Then, footprint was obtained by using imprint material, with the subject standing in a re- laxed position and using the AutoCAD computer program, the length of the foot, excluding the toes, was measured the perpendicular distance from the metatarsophalangel joint to the most posterior aspects of the heel (Cowan and Jones, 1993). The NNHt was then calculated by dividing the navicular tuberosity height by the truncated foot length, with a lower normalized navicular height ratio indicating a flatter foot.

\section{Procedures}

The navicular height of the subjects was measured and the NDp was performed three times each by using a digital height gauge. After a break, the NDt was performed in the sitting position and in the relaxed stance position. With the subjects standing in a relaxed position, we measured the FPI, and the DAHR was measured by using a ruler. Finally, after the feet of all subjects were imprinted, the $\mathrm{AI}$ and the NNHt were calculated by using the AutoCAD computer program.

\section{Statistical Analysis}

All analyses were performed using the SPSS ver. 12.0 program. ANOVA analysis was used for the variance of difference between the NDp test and five kinds of clinical measurements, and a post-hoc analysis was performed by using the Tukey test. The level of significance was set at $a=.05$.

Table 2. A comparison of NDt, FPI, AI, DAHR, NNHt, among pronated foot, normal foot, and supinated foot

\begin{tabular}{|c|c|c|c|c|}
\hline & $\begin{array}{l}\text { Pronated foot } \\
\qquad(\mathrm{n}=22)\end{array}$ & $\begin{array}{l}\text { Normal foot } \\
\qquad(\mathrm{n}=27)\end{array}$ & $\begin{array}{l}\text { Supinated foot } \\
\qquad(\mathrm{n}=15)\end{array}$ & $\mathrm{F}$ \\
\hline $\mathrm{NDt}^{\mathrm{d}}(\mathrm{mm})$ & $.75 \pm .21^{\mathrm{a}}$ & $.45 \pm .13^{\mathrm{b}}$ & $.34 \pm .13^{\mathrm{b}}$ & $34.101^{*}$ \\
\hline $\mathrm{FPI}^{\mathrm{e}}$ & $8.08 \pm 1.77$ & $3.72 \pm 1.88^{\mathrm{b}}$ & $-4.13 \pm 3.74^{\mathrm{bc}}$ & $115.130^{*}$ \\
\hline $\mathrm{AI}^{\mathrm{f}}$ & $.27 \pm .03$ & $.24 \pm .03$ & $.22 \pm .03^{\mathrm{b}}$ & $14.153^{*}$ \\
\hline $\mathrm{DAHR}^{\mathrm{g}}$ & $.31 \pm .03$ & $.33 \pm .03$ & $.35 \pm .03^{\mathrm{b}}$ & $10.544^{*}$ \\
\hline $\mathrm{NNHt}^{\mathrm{h}}$ & $.24 \pm .22$ & $.28 \pm .03^{\mathrm{b}}$ & $.32 \pm .03^{\mathrm{bc}}$ & $33.343^{*}$ \\
\hline
\end{tabular}

amean \pm standard deviation, ${ }^{b}$ significant difference as compared with pronated foot, ${ }^{\mathrm{c}}$ significant difference as compared with normal foot, ${ }^{\mathrm{d}}$ navicular drift test, ${ }^{\mathrm{e}}$ foot posture index, ${ }^{\mathrm{f}}$ arch index, ${ }^{\mathrm{g}}$ dorsal arch height ratio, ${ }^{\mathrm{h}}$ normalized navicular height truncated, ${ }^{*} \mathrm{p}<.05$. 


\section{Results}

Difference of measurements among NDt, FPI, AI, DAHR, and NNHt according to the foot types

There was a significant difference in the five kinds of clinical measurements according to foot posture types $(\mathrm{p}<.05)$. In the post-hoc analysis, both FPI and NNHt showed significant differences in all foot postures. However, with AI and DAHR, differences only existed between the pronated foot and the supinated foot, while with $\mathrm{NDt}$, there were significant differences between the pronated foot and normal foot and between the supinated foot and the pronated foot $(\mathrm{p}<.05)$ (Table 2$)$.

\section{Discussion}

The foot is the most distal segmental area of the lower extremities, and it is a small base of support upon which the body maintains balance. Although minor biomechanical alterations of foot in the support surface foot may influence the posture control and balance of the body, general measurements that evaluate foot posture lack. In this study, after classifying foot posture by the NDp, which has been widely used to assess foot posture, the most proper method of distinguishing three types of feet, which are classified by five clinical measurements, would be suggested, and finally, the most effective clinical measurement will be discovered. The results of this study showed significant differences among five clinical measurements according to foot posture types. In the post-hoc analysis, the FPI and the NNHt showed significant differences in three foot types $(\mathrm{p}<.05)$.

The results of this study show that the mean value of the horizontal movement of the navicular is $5.2 \pm 2.3$, which is somewhat lower than that reported by Vinicombe et al (2001). Considering that most of the subjects of this study were women and that they were apt to choose smaller size shoes, the results of the study coincide with the study by Menz (1998), which claimed that foot size is important for $\mathrm{de}^{-}$ termining navicular drop pronation values. The amount of horizontal movement of the navicular in the pronated foot group, which was determined $\mathrm{ac}^{-}$ cording to the $\mathrm{NDp}$, is relatively larger than that of the supinated foot group. It demonstrates that the amount of horizontal movement and vertical movement of the navicular is related with the foot postures.

Although the FPI is an observational tool in many ways, it is not a proper method for measuring arch height. However, it has the advantage that it provides all the characteristics of foot posture in measuring the MLA and that it is easy to measure without using specialized equipment. The FPI is strongly affected by the shape of the soft tissue and provides limited information about the bony structures of the MLA (Powers et al, 1995). Compared with Redmond's classification of foot posture, the FPI score of this study was showed a slightly different scale: pronated (5 to 11), normal (0 to 7 ), supinated $(-9$ to -1$)$. The difference between these values is due to the degree of hip rotation when the subjects were in a relaxed standing position.

The AI is a reliable tool that quantifies foot characteristics based on a static footprint and does not rely on the clinical experience of the examiner (Levinger et $\mathrm{al}, 2010)$. The AI values obtained in this study (mean value \pm standard deviation, .24 \pm .03 ) are similar to those of previous studies (.25 .04$)$ (Menz and Munteanu, 2005) despite the fact that subjects' generalized characteristics were different. Cavangh and Rodgers (1987) classified AI values as the normal values from .22 to .25; values $<.21 \mathrm{in}^{-}$ dicated a varus foot (supinated foot), and values $>.26$ indicated a valgus foot (pronated foot). The results of this study are as follows: the normal value is 24 , the pronated value is .27 , and the supinated value is .22. These values are similar to those of previous studies. Billis et al (2007) reported that correlations between $\mathrm{AI}$ and the $\mathrm{NDp}$ and the NDt were low in both bipedal and a single-leg stances. They also suggested these findings could partly be attributed to 
the large variability in body fat percentages. However, this study showed that the AI measurement is significantly different from the NDp for the classification of foot posture, and the AI values of overweight subjects were also higher regardless of foot posture. The weight of subjects could be composed of factor in $\mathrm{AI}$ value.

McPoil et al (2008) have described the DAHR as a method of considering the disadvantages of the NDp, which depends on the examiner's experience. Also, McPoil et al (2009) noted that the greater the vertical change in dorsal arch height is, the greater the amount of foot mobility there is. The mean values of this study, which are in accord with the DAHR, are slightly higher than those of previous research (Williams and McClay, 2000). The subjects in this study, as compared to that of Williams and McClay (2000), were mostly women. Considering that women's foot lengths and the average foot length in this study were short, we speculate that there are the differences in the DAHR values because of difference of foot length.

The NNHt measurement was reported to be the most valid measurement in Williams and McClay's (2000) research. In terms of the results of this study, the mean value of $\mathrm{NNHt}(.27 \pm .03)$ was relatively larger than that of Williams and McClay (2000)

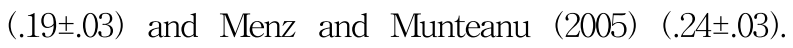
Williams (2000) measured the value of NNHt in a 90\% weight-bearing posture, and Menz and Munteanu (2005) measured the value of NNHt of elderly patients with osteoarthritis (OA) who were relatively overweight, from $73.14 \pm 15.49$ for the control group to $85.13 \pm 13.67$ for the experimental group. We think these differences in measurement caused the difference in the mean values. Also, while Williams and McClay (2000) measured the foot length, excluding the toes, with a ruler, we took a foot print and calculated the value of NNHt by using the AutoCAD computer program, which would also add to the difference in the mean value.

In this study, we have tried to discover the most effective measurement among the five clinical measurements according to the classification of foot posture by NDp. All five clinical measurements showed significant differences in evaluating static foot posture. Particularly, in post-hoc analysis, FPI and NNHt showed a significant difference. However, with $\mathrm{NDt}$, there were significant differences between the pronated foot and the normal foot and between the supinated foot and the pronated foot. With AI and DAHR, the differences were only between the pronated foot and the supinated foot. These results show that FPI and NNHt are effective measurements in evaluating foot posture. As NNHt is somewhat time-consuming and does not have generalized data, it is difficult to classify normal and abnormal feet. In contrast, FPI and NDt require minimal equipment and time, and they are the simplest methods to perform in a clinic.

This study has a few limitations, the first of which is that the number of subjects is relatively small. 64 feet were measured at random, but there was a relatively smaller number of supinated feet. Secondly, this study was performed on subjects with healthy lower limbs. Systemic conditions, such as diabetes and rhematoid arthritis, or OA can change foot posture and limit the mobility. This limitation can't bring about useful results in a clinic. Thirdly, all measurements were performed in a static position. Thus, future studies should be performed to find out the most effective clinical measurement for those who have problems with feet or lower limbs, and they should also consider dynamic foot positions.

\section{Conclusion}

In this study, we attempted to discover the most effective measurement among five kinds of clinical measurements in a static position according to the classification of foot types by the NDp. As a result, FPI and NNHt showed a significant difference $(\mathrm{p}<.05)$ regarding every foot posture. AI and DAHR 
showed significant differences $(\mathrm{p}<.05)$ only between pronated feet and the supinated feet in post-hoc analysis. These results showed that FPI and NNHt, as well as NDp are proper measurements in $\mathrm{de}^{-}$ termining foot types. Therefore, it could be possible to assess correct and objective foot posture by using FPI and NNHt.

\section{References}

Allen MK, Glasoe WM. Metrocom measurement of navicular drop in subjects with anterior cruciate ligament injury. J Athl Train. 2000;35(4):403-406.

Albensi RJ, Nyland J, Caborn DN. The relationship of body weight and clinical foot and ankle measurements to the heel forces of forward and backward walking. J Athl Train. 1999;34(4): 328-333.

Bennett JE, Reinking MF, Pluemer B, et al. Factors contributing to the development of medial tibial stress syndrome in high school runners. J Orthop Sports Phys Ther. 2001;31(9):504-510.

Billis E, Katsakiori E, Kapodistrias C, et al. Assessment of foot posture: Correlation between different clinical techniques. Foot. 2007;17(2): 65-72.

Brody DM. Techniques in the evaluation and treatment of the injured runner. Orthop Clin North Am. 1982;13(3):541-558.

Brukner PD. Clinical Sports Medicine. McGraw Hill, 1996:51-52.

Cavanagh PR, Rodgers MM. The arch index: A useful measure from footprints. J Biomech. 1987;20(5):547-551.

Cowan DN, Jones BH, Robinson JR. Foot morphologic characteristics and risk of exercise-related injury. Arch Fam Med. 1993;2(7):773-777.

Cowan DN, Robinson JR, Jones BH, et al. Consistency of visual assessments of arch height among clinicians. Foot Ankle Int. 1994;15(4):213-217.
Cornwall MW, McPoil TG. Relative movement of the navicular bone during normal walking. Foot Ankle Int. 1999;20(8):507-512.

Cote KP, Brunet ME, Gansneder BM, et al. Effects of pronated and supinated foot postures on static and dynamic postural stability. J Athl Train. 2005;40(1):41-46

Hawes MR, Nachbauer W, Sovak D, et al. Footprint parameters as a measure of arch height. Foot Ankle. 1992;13(1):22-26.

Hertel J, Gay MR, Denegar CR. Differences in postural control during single-leg stance among healthy individuals with different foot types. J Athl Train. 2002;37(2):129-132.

Inman VT. The influence of the foot - ankle complex on the proximal skeletal structures. Artif Limbs. 1969;13(1):59-65.

Levinger P, Menz HB, Fotoohabadi MR, et al. Foot posture in people with medial compartment knee osteoarthritis. J Foot Ankle Res. 2010;3:29-36.

McCrory JL, Young MJ, Boulton AJM, et al. Arch index as a predictor of arch height. Foot. 1997;7(2):79-81.

McPoil TG, Cornwall MW, Vicenzino B, et al. Effect of using truncated versus total foot length to calculate the arch height ratio. Foot. 2008;18(4):220-227.

McPoil TG, Vicenzino B, Cornwall MW, et al. Reliability and normative values for the foot mobility magnitude: A composite measure of vertical and medial-lateral mobility of the midfoot. J Foot Ankle Res. 2009;2:6.

Menz HB. Alternative techniques for the clinical as ${ }^{-}$ sessment of foot pronation. J Am Podiatr Med Assoc. 1998;88(3):119-129.

Menz HB. Clinical hindfoot measurement: A critical review of the literature. Foot. 1995;5(2):57-64.

Menz HB, Munteanu SE. Validity of 3 clinical techniques for the measurement of static foot posture in older people. J Orthop Sports Phys Ther. 2005;35(8):479-486.

Morrison SC, Ferrari J. Inter-rater reliability of the 
Foot Posture Index (FPI-6) in the assessment of the paediatric foot. J Foot Ankle Res. 2009;2:26.

Mueller MJ, Host JV, Norton BJ. Navicular drop as a composite measure of excessive pronation. J Am Podiatr Med Assoc. 1993;83(4):198-202.

Nigg BM, Cole GK, Nachbauer W. Effects of arch height of the foot on angular motion of the lower extremities in running. $\mathrm{J}$ Biomech. 1993;26(8):909-916.

Nigg BM, Fisher V, Allinger TL, et al. Range of motion of the foot as a function of age. Foot Ankle. 1992;13(6):336-343.

Plisky MS, Rauh MJ, Heiderscheit B, et al. Medial tibial stress syndrome in high school cross-country runners: Incidence and risk factors. J Orthop Sports Phys Ther. 2007;37(2): 40-47.

Powers CM, Maffucci R, Hampton S. Rearfoot posture in subjects with patellofemoral pain. J Orthop Sports Phys Ther. 1995;22(4):155-160.

Redmond AC, Crosbie J, Ouvrier RA. Development and validation of a novel rating system for scoring standing foot posture: Foot posture Index. Clin Biomech (Bristol, Avon). 2006;21(1) :89-98.

Reilly K, Barker K, Shamley D, et al. The role of foot and ankle assessment of patients with lower limb osteoarthritis. Physiotherapy. 2009;95(3): 164-169.
Sahrmann SA. Movement System Impairment Syndromes of the Extremities, Cervical and Thoracic Spines. St. Louis, MO, Mosby Inc., 2011:439-480.

Sell KE, Verity TM, Worrell TW, et al. Two measurement techniques for assessing subtalar joint position: A reliability study. J Orthop Sports Phys Ther. 1994;19(3):162-167.

Scharfbillig R, Evans AM, Copper AW, et al. Criterion validation of four criteria of the foot posture index. J Am Podiatr Med Assoc. 2004;94(1):31-38.

Shrader JA, Popovich JM Jr, Gracey GC, et al. Navicular drop measurement in people with rheumatoid arthritis: Interrater and intrarater reliability. Phys Ther. 2005;85(7):656-664.

Vinicombe A, Raspovic A, Menz HB. Reliability of navicular displacement measurement as a clinical indicator of foot posture. J Am Podiatr Med Assoc. 2001;91(5):262-268.

Williams DS, McClay IS. Measurements used to characterize the foot and the medial longitudinal arch: Reliability and validity. Phys Ther. 2000;80(9):864-871.

This article was received May 7, 2012, was $\mathrm{re}^{-}$ viewed May 7, 2012, and was accepted August 8, 2012. 